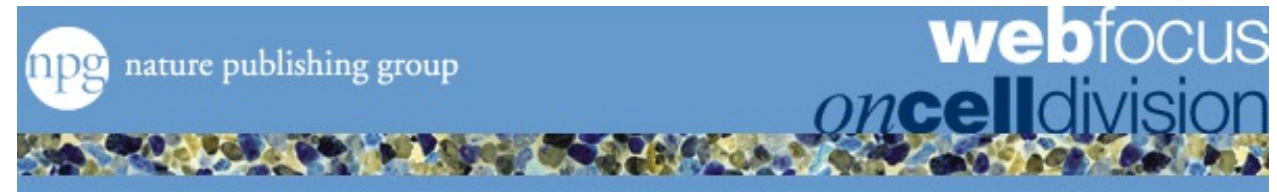

Milestone 8

\title{
Do not pass G0
}

In the early 1970s, the nature and duration of the G0 phase were a source of controversy - indeed, some had even proposed that GO did not really exist. In 1974, however, reporting in Proceedings of the National Academy of Sciences, Arthur Pardee argued that cells do go into a G0 state, and that this is an equivalent state irrespective of the means by which it is induced. He showed that cells re-enter the cell cycle by transiting a 'restriction point' - after which they are committed to the cell cycle - and argued that this switch is defective in cancer cells.

Pardee set out to discover whether cells switch between the quiescent and proliferative states at one or several points in the cell cycle. To do this, he exposed a population of Syrian hamster BHK cells to various blocking agents - such as high levels of cyclic AMP or starvation of either nutrients or serum. He then restored optimal growth conditions and measured the kinetics of $\left[{ }^{3} \mathrm{H}\right]$ thymidine incorporation (indicating escape from the block). He found that after five different blocks, the quiescent cells required the same amount of time to recommence DNA synthesis. Transformed cells, by contrast, did not recover their ability to incorporate thymidine, indicating a different response to the block.

But Pardee didn't stop there. He recognized that these experiments had several limitations - the main problem being the assessment of exactly when DNA synthesis was re-initiated. So, rather than using just a single block, Pardee imposed one block, then shifted cells to a different type of block and asked whether they could proceed to DNA synthesis. As he explained, "if the block applied second stops cells at an earlier time in G1 than does the block applied first, the cells should make DNA. But if the block supplied second acts later or at the same point as the one applied earlier, the cells should not progress on to DNA synthesis".

Pardee observed that, in every case, the second block prevented re-initiation of DNA synthesis, indicating that all blocks acted at more or less the same point. He further tested this conclusion with a series of three-step experiments, where thymidine uptake was measured during the last treatment. Again, the cells always seemed to be blocked at a point between the M- and S-phases - that is, in G1. Pardee then showed that when cells are treated with colchicine and hydroxyurea non-physiological agents that stop cells in $M$ and $S$ respectively - they do not stop at this point.

Pardee termed this switching point the 'restriction point', and proposed that it "permit[s] normal cells to retain viability by a shift to minimal metabolism upon differentiation... when conditions are suboptimal for growth". Eleven years later, in a seminal paper, Zetterberg and Larsson followed the behaviour of individual cells by time-lapse microscopy to provide a detailed map of cell responses to serum starvation, cyclohexamide treatment or exposure to particular growth factors. They showed that a variable growth-factor-dependent phase preceded a growth-factorindependent phase.

Now, almost 30 years on from Pardee's proposal of the restriction point, the intricate network of cyclin-dependent kinases that control commitment to the cell cycle show how clearly his view encapsulates the way we think about commitment to the cell cycle during passage through an unperturbed $\mathrm{G} 1$ or exit from $\mathrm{G} 0$.

\section{Alison Mitchell, Editor, Nature Reviews Molecular Cell Biology}

\section{References}

\section{ORIGINAL RESEARCH PAPER}

Pardee, A. B. A restriction point for control of normal animal cell proliferation. Proc. Natl Acad. Sci. USA 71, 1286-1290 (1974)

Zetterberg, A. \& Larsson, O. Kinetic analysis of regulatory events in G1 leading to proliferation or quiescence of Swiss $3 T 3$ cells. Proc. Natl Acad. Sci USA 82, 53655369 (1985)

Pardee, A. B. G1 events and regulation of cell proliferation. Science 246, 603-608 (1989) 